$01,05,17$

\title{
Лазерно-индуцированная генерация поверхностных периодических структур в средах с нелинейной диффузией
}

\author{
() В.М. Журавлев, И.О. Золотовский , Д.А. Коробко, В.М. Морозов, \\ В.В. Светухин, И.О. Явтушенко, М.С. Явтушенко
}

Ульяновский государственный университет, Ульяновск, Россия

I E-mail: rafzol.14@mail.ru

(Поступила в Редакцию 31 января 2017 г.

В окончательной редакции 28 апреля 2017 г.)

\begin{abstract}
Предложена модель быстрого формирования высококонтрастной периодической структуры, возникающей на поверхности полупроводника под действием лазерного излучения. В рамках модели среды с нелинейной диффузией неравновесных носителей (дефектов) рассмотрен процесс вырастания поверхностной структуры за счет взаимодействия поверхностных плазмон-поляритонов, возбуждаемых на неравновесных электронах, с падающим лазерным излучением. Предсказан резонансный эффект сверхбыстрого пико- и субпикосекундного усиления генерируемой на поверхности плазмон-поляритонной структуры, при реализации которого может быть получена высококонтрастная решетка дефектов.
\end{abstract}

Работа выполнена при поддержке Министерства образования и науки Российской Федерации (проект № 14.Z50.31.0015) и Российского фонда фундаментальных исследований (проект № 16-42-730398).

DOI: 10.21883/FTT.2017.12.45222.026

\section{1. Введение}

Эффекты, связанные с образованием на поверхности металлов и полупроводников пространственно-модулированных структур с периодом как порядка длины световой волны [1-4], так и значительно меньшей [5-10], привлекают значительный интерес исследователей по причине возможности их использования в технологиях фотоники, интегральной оптики, а также микро- и наноэлектроники [11-13]. Отдельный интерес может представлять формирование субволновых высококонтрастных структур на поверхности полупроводников [10-15].

Современные представления о формировании когерентных периодических структур на поверхности полупроводников сводятся к следующему. При облучении ультракороткими импульсами (длительностью менее $100 \mathrm{fs})$ кристаллическая решетка остается „холодной“ и практически не принимает участия в поглощении лазерного излучения [8-10]. Последнее поглощается электронной подрешеткой и вызывает образование на поверхности полупроводника тонкой пленки металлизации [3-5]. Процесс такой металлизации поверхности полупроводника является нетермическим фазовым переходом, инициированным световым излучением. Если под действием лазерного излучения на поверхности или в объеме полупроводника формируется металлизированный слой, то при выполнении определенного условия на границе „металлизированная поверхностьсреда“ возможна реализация условий для возбуждения на неравновесных электронах поверхностных плазмонполяритонов (ППП), взаимодействующих с падающим лазерным излучением. Известно, что в результате этого взаимодействия на поверхности облучаемого материала формируется периодическая структура, модулированная световым полем, с периодом порядка либо значительно меньшим центральной длины волны источника [14-16].

Процесс возбуждения ППП на поверхности полупроводника в лазерном поле заслуживает отдельного рассмотрения. Падающая волна взаимодействует с неоднородностями реальной поверхности, являющейся шероховатой, с микронеровностями. В пространстве вблизи поверхности формируется периодически модулированное волновое поле, возникающее в результате интерференции падающей световой волны и волны, рассеяной такой поверхностью. Среди рассеянных на шероховатостях волн имеются как зеркально отраженные компоненты падающей волны, так и компоненты, дифрагировавшие на различных участках поверхности. Так как всякую шероховатую поверхность можно представить в виде комбинации гармонических решеток со случайными ориентациями штрихов, случайными периодами и амплитудами рельефа, (т.е. разложить ее в пространственный спектр Фурье), то рассеяние падающей волны на шероховатостях можно рассматривать как дифракцию на различных Фурье-компонентах спектра шероховатости. Таким образом, возникает интерференция падающей волны с определенными (резонансными) компонентами дифрагированного поля, соответствующими возникновению на поверхности тонкой пленки локализованного ППП. Соответствующий механизм образования ППП был рассмотрен в работах $[1,17,18]$.

В ряде случаев может наблюдаться исключительно высокая контрастность образующихся периодических структур, которая представляет особый интерес. Столь 
высокая контрастность не может быть объяснена, с нашей точки зрения, действием только периодически модулированного интерференционного поля лазерного излучения.

В настоящей работе для объяснения эффекта генерации высококонтрастных структур с относительно большой амплитудой рельефа предлагается модель, связанная $\mathrm{c}$ нелинейной зависимостью коэффициента диффузии от концентрации неравновесных носителей, образующих металлизированную пленку на поверхности полупроводника.

В общем случае различные модели, связанные с влиянием нелинейной диффузии, исследовались достаточно давно, особенно в связи с задачами распространения тепла в средах с нелинейными коэффициентами теплопроводности [19-21]. Соответствующие модели представляют собой прямой аналог диффузионных уравнений для концентраций дефектов. От этих обобщенных моделей диффузии дефектов перейдем к частной модели, описывающей нелинейную диффузию неравновесных носителей заряда в поле лазерного излучения, за счет введения в общие уравнения дополнительных условий применительно к нашей задаче. В предлагаемой нами модели коэффициент диффузии дефектов связан с концентрацией неравновесных носителей зарядов, образующихся на поверхности облучаемого полупроводника, с помощью соотношения

$$
D=\frac{D_{0}}{n+n_{0}}
$$

где $D_{0}=D_{p} n_{0}, n-$ концентрация генерируемых дефектов (неравновесных носителей), $\left[\mathrm{cm}^{-3}\right] ; D_{p}-$ собственно коэффициент диффузии дефектов (неравновесных носителей), $\left[\mathrm{cm}^{2} / \mathrm{s}\right]$, а параметр $n_{0}$ характеризует влияние концентрации неравновесных носителей на диффузионные процессы. В том случае, когда $n / n_{0} \rightarrow 0$, мы переходим к „классической“ модели диффузии.

\section{2. Уравнение нелинейной диффузии}

Используя диффузионный коэффициент вида (1), можно записать общее уравнение диффузии дефектов в следующем виде [22-24]:

$$
\frac{\partial n}{\partial t}=\frac{\partial}{\partial z}\left(\frac{D_{0}}{n+n_{0}} \frac{\partial n}{\partial z}\right)-\mu n+G,
$$

где $t-$ время, $z$ - поперечная координата, $\mu-$ скорость рекомбинации дефектов, $G$ - скорость генерации дефектов. Вводя функцию $\xi=n+n_{0}$, преобразуем это уравнение к следующему виду:

$$
\frac{\partial \xi}{\partial t}=\frac{\partial}{\partial z}\left(\frac{D_{0}}{\xi} \frac{\partial \xi}{\partial z}\right)-\mu \xi+G_{e f}
$$

Здесь введен эффективный коэффициент генерации дефектов

$$
G_{e f}=G+\mu n_{0} .
$$

Далее, переходя к нормированной координате $x=z / \sqrt{D_{0}}$, получаем уравнение:

$$
\frac{\partial \xi}{\partial t}=\frac{\partial^{2} \ln \xi}{\partial x^{2}}-\mu \xi+G_{e f}
$$

\section{3. Отрицательная эффективная диффузия. Дифффузионный „взрыв“}

При переходе от дефектов к их частному случаю локальных неоднородностей распределения неравновесных носителей заряда нужно ввести ряд уточняющих пояснений. В рассматриваемой нами модели за счет взаимодействия падающей волны с образующимися на стартовых неровностях рельефа поверхности ППП [25-31] происходит быстрое формирование поверхностного поля, обеспечивающего генерацию неравновесных носителей заряда. Характерные времена составляют значения порядка субпикосекунд, а характерные длины соотвествуют глубине проникновения излучения и периоду плазмон-поляритонной структуры (ППС), т.е. в общем случае менее $1 \mu \mathrm{m}$. Поскольку диффузионная длина существенно больше характерных длин, то можно предполагать, что, наряду с быстрыми процессами (формирования интерференционного поля падающего лазерного импульса и ППП), в самой среде происходят относительно медленные процессы формирования структур - образование контрастного рельефа на поверхности. Для выявления условий возникновения длинноволновых возмущений за счет тех или иных неустойчивостей можно воспользоваться методом многомасштабных разложений [19-21], позволяющим выявить растущие при данных условиях моды.

Для анализа полученного уравнения (4) используем метод решения уравнения в медленных переменных. Это означает, что, наряду со стандартными единичными масштабами времени, ответственными за переходные процессы в системе, решение уравнения (4) будет содержать зависимость от медленных переменных времени $T=\varepsilon t$ и длины $X=\sqrt{\varepsilon} x[19,20]$. Здесь $\varepsilon-$ безразмерный малый параметр, характеризующий время возникновения структуры по отношению к масштабу времени $\tau$. При этом безразмерный масштаб длины возникающей структуры определяется малой величиной $\sqrt{\varepsilon}$. Зависимость функции $\xi$ от координат и времени можно записать в следующем виде:

$$
\xi=\xi(x, t)=\chi(t, X, T, \varepsilon) .
$$

Представим функцию $\xi(x, t)$ в виде ряда по малому параметру $\varepsilon$ :

$$
\chi(t, X, T, \varepsilon)=\xi_{0}(t, X, T)+\xi_{1}(t, X, T) \varepsilon+O\left(\varepsilon^{2}\right) .
$$

С использованием стандартных для метода многомасштабных разложений соотношений

$$
\frac{\partial \xi}{\partial t}=\frac{\partial \chi}{\partial t}+\varepsilon \frac{\partial \chi}{\partial T}, \quad \frac{\partial \xi}{\partial x}=\sqrt{\varepsilon} \frac{\partial \chi}{\partial X}
$$


уравнение (4) преобразуется к виду

$$
\chi_{t}=-\mu \chi+G_{e f}-\varepsilon \frac{\partial \xi}{\partial T}+\varepsilon \frac{\partial^{2} \chi}{\partial X^{2}} .
$$

Коэффициенты разложения (6) удовлетворяют следующим уравнениям:

$$
\xi_{0, t}=-\mu \xi_{0}+g, \quad \xi_{1, t}=-\mu \xi_{1}-\frac{\partial \xi_{0}}{\partial T}+\frac{\partial^{2} \ln \xi_{0}}{\partial X^{2}} .
$$

Решение в нулевом порядке принимает вид

$$
\xi_{0}=C(X, T) e^{-\mu}+G_{e f} / \mu,
$$

где $C(X, T)$ - постоянная интегрирования, зависящая в данном случае от медленных переменных. Аналогично решение в первом порядке можно представить как

$$
\xi_{1}=C_{1}(X, T) e^{-\mu t}+e^{-\mu t} \int_{0}^{t} e^{\mu t^{\prime}}\left[-\frac{\partial \xi_{0}}{\partial T}+\frac{\partial^{2} \ln \xi_{0}}{\partial X^{2}}\right] d t^{\prime}
$$

где $C_{1}(X, T)$ - постоянная интегрирования, также зависящая от медленных переменных.

Для нахождения решения в первом порядке вычисляем интегралы в (11)

$$
\begin{gathered}
I_{1}=-\int_{0}^{t} e^{\mu t^{\prime}} \frac{\partial \xi_{0}}{\partial T} d t^{\prime}=-\frac{\partial C}{\partial T} t, \\
I_{2}=\int_{0}^{t} e^{\mu t^{\prime}} \frac{\partial^{2} \ln \xi_{0}}{\partial X^{2}} d t^{\prime} \\
=\int_{0}^{t} e^{\mu t^{\prime}} \frac{\left(C e^{-\mu t^{\prime}}+G_{e f}\right) C_{X X} e^{-\mu t^{\prime}}-C_{X}^{2} e^{-2 \mu t^{\prime}}}{\left(C e^{-\mu t^{\prime}}+G_{e f} / \mu\right)^{2}} d t^{\prime} \\
=C_{X X} \frac{\mu}{G_{e f}}\left(t+\frac{1}{\mu} \ln \left(C e^{-\mu t}+G_{e f} / \mu\right)\right) \\
-\frac{C_{X}^{2}}{\mu C\left(C e^{-\mu t}+G_{e f} / \mu\right)} .
\end{gathered}
$$

В (13) введены обозначения: $C_{X}=\partial C / \partial X$ и $C_{X X}=\partial^{2} C / \partial X^{2}$. Интегралы $I_{1}$ и $I_{2}$ содержат слагаемые, растущие в асимптотике при $t \rightarrow \infty$ по линейному закону. Согласно стандартной процедуре исключения резонансов, необходимо потребовать, чтобы эти слагаемые обратились в ноль. В интеграле $I_{2}$ при $t \rightarrow \infty$ слагаемые, содержащие множитель $t$, взаимно сокращаются. Поэтому функция $C(X, T)$, определяющая динамику поля дефектов, удовлетворяет уравнению

$$
\frac{\partial C}{\partial T}-\frac{\mu}{G_{e f}} \frac{\partial^{2} C}{\partial X^{2}}=0
$$

Таким образом, получено уравнение, определяющее динамику генерируемых дефектов в поле излучения, которое можно назвать дифффузионным уравнением с эффективной диффузией $D_{e f}=\mu / G_{e f}$.

Самым интересным следствием уравнения (14) является возможность „возникновения“ отрицательной эффективной диффузии, когда $\mu / G_{e f}<0$. Режим отрицательной диффузии может быть реализован в двух случаях

$$
\mu<0 \text { и } G_{e f}>0\left(\text { т. е. } G+\mu n_{0}>0\right)
$$

или

$$
\left.\mu>0 \text { и } G_{e f}<0 \text { (т.е. } G+\mu n_{0}<0\right) \text {. }
$$

Второй случай, очевидно, соответствует быстрой рекомбинации соответствующих дефектов вплоть до нулевого значения за ограниченный промежуток времени и, скорее всего, не может представлять существенный интерес для разного рода практических применений.

Очевидно, что наибольший интерес для нас будет представлять первый случай, соответстветствующий экспоненциально нарастающей генерации дефектов.

Уравнение (14) представляет собой параболическое уравнение в медленных переменных с эффективным коэффициентом диффузии, равным $\mu / G_{e f}$, который меняет знак в зависимости от знака $g$. Пространственнопериодические решения являются суперпозицией решений вида [32]

$$
C(X, T)=e^{p T}(A \cos (k X)+B \sin (k X)),
$$

где параметр $p$ связан с волновым числом $k$ по медленной координате $X$ соотношением $p=-\frac{k^{2} \mu}{G_{e f}}$. Таким образом, переходя к нашему частному случаю, период формирующейся дифракционной решетки (пространственной неоднородности), реализующей условие фазового синхронизма между падаюшей волной и формирующегося ППП в нашем случае определяется соотношением

$$
k_{0} \sin \theta=k_{t} \cong q-2 \pi / d,
$$

где $d-$ пространственный период формируемой решетки; $d \approx 2 \pi /\left(q-k_{t}\right)$. С учетом неравенства $k_{0} \ll q$ с хорошей степенью точности можно считать, что $d \approx 2 \pi / q$, величина которого составляет $d \sim 0.01-10 \mu \mathrm{m}[1,25-32]$.

Подставляя (16) в (14) и возвращаясь в „исходные координаты“ времени и длины, находим

$$
\begin{aligned}
C(x, t) & =e^{(p \varepsilon-\mu) t}(A \cos (k \sqrt{\varepsilon} x)+B \sin (k \sqrt{\varepsilon} x)) \\
& =e^{g t}(A \cos (q z)+B \sin (q z)),
\end{aligned}
$$

где $q=2 \pi / d$.

В данном случае с хорошей степенью точности можно считать, что волновое число ППП, возбуждаемого на индуцированной металлической пленке толщины $h$, равно $q \cong \omega / v_{f}$, где $v_{f}-$ фазовая скорость соответствующего плазмон-поляритона. 


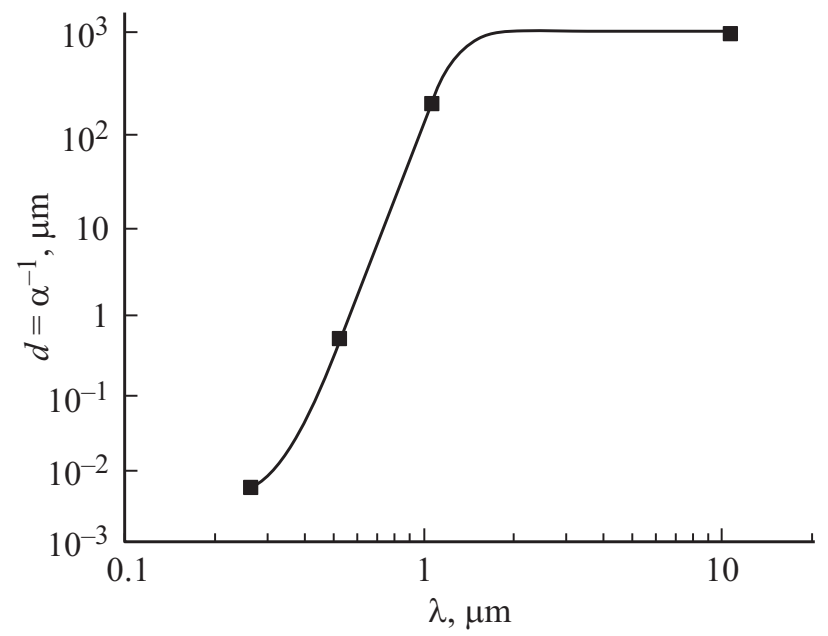

Рис. 1. Зависимость глубины проникновения лазерного излучения от длины волны излучения для кремния. Данные взяты из [17] и представлены в таблице.

Толщина индуцированного лазерным излучением металлизированного слоя для полупроводника определяется простым соотношением $h \approx 1 / \alpha$, где $\alpha$ - линейный коэффициент поглощения полупроводника, обеспечивающий генерацию неравновесных зарядов и изменяющийся в исключительно широком интервале значений в зависимости от длины волны лазерного источника. Значения параметров в зависимости от длин волн лазерного излучения для кристаллического кремния приведены в таблице [17]. На рис. 1 представлена зависимость глубины проникновения излучения для кремния для разных длин волн.

На основании результатов работ [23-28,33-43] с хорошей степенью точности получаем дисперсионное соотношение для константы распространения $q$ формируемого на поверхности плазмон-поляритона

$$
\begin{aligned}
\exp \left(-2 \theta_{2} h\right) & \approx \exp \left(-2 \theta_{2} / \alpha\right) \\
& \approx\left(\frac{\theta_{2} \varepsilon_{1}+\theta_{1} \varepsilon_{2}}{\theta_{2} \varepsilon_{1}-\theta_{1} \varepsilon_{2}}\right)\left(\frac{\theta_{2} \varepsilon_{3}+\theta_{3} \varepsilon_{2}}{\theta_{2} \varepsilon_{3}-\theta_{3} \varepsilon_{2}}\right) .
\end{aligned}
$$

Здесь $\theta_{j}=\sqrt{q^{2}-k_{0}^{2} \varepsilon_{j}}(j=1,2,3)$ - постоянная затухания $z$-составляющей мнимого волнового вектора в проводящем слое и диэлектриках, $k_{0}=\omega / c, c-$ скорость света в вакууме, $\varepsilon_{j}$ - действительные составляющие рассматриваемых сред соответственно. При этом

Параметры поглощения кремния при облучении различными лазерными источниками [17]

\begin{tabular}{c|c|c|c|c}
\hline $\begin{array}{c}\text { Длина волны } \\
\text { излучения, } \mu \mathrm{m}\end{array}$ & 0.265 & 0.53 & 1.06 & 10.6 \\
\hline $\begin{array}{l}\text { Параметр } \\
\text { поглощения, } d=\alpha^{-1}\end{array}$ & $6 \mathrm{~nm}$ & $500 \mathrm{~nm}$ & $200 \mu \mathrm{m}$ & $1 \mathrm{~mm}$ \\
\hline
\end{tabular}

первая среда предполагается вакуумом и соответственно $\varepsilon_{1}=1$; вторая среда - индуцированная пленка металлизации, образуемая неравновесными зарядами, для которой верно $\varepsilon_{2} \approx 1-\omega_{p}^{2} / \omega^{2}$, где $\omega_{p}=\sqrt{4 \pi n_{e} e^{2} / m_{e}}-$ плазменная частота неравновесной плазмы на поверхности полупроводника, $n_{e} \sim 10^{20} \mathrm{~cm}^{-3}-$ концентрация зарядов в металлизированной пленке [17], e и $m_{e}$ - заряд и масса электрона соответственно; третья среда собственно облучаемый лазером полупроводник с диэлектрической проницаемостью $\varepsilon_{3}$ (см. рис. 2).

Для эффективного инкремента усиления периодических возмущений при реализуемой отрицательной эфективной диффузии верно соотношение

$$
\begin{aligned}
g & =p \varepsilon-\mu=-\frac{q^{2} \mu D_{0}}{G_{e f}}-\mu=-\mu\left(1+\frac{q^{2} D_{0}}{G_{e f}}\right) \\
& =-\mu\left(1+\frac{q^{2} n_{0} D_{p}}{G_{e f}}\right)=-\mu+g_{D} .
\end{aligned}
$$

Приведем оценку характерных масштабов длины и времени для различных процессов в рассматриваемой нелинейной системе.

Для задач, связанных с лазерным облучением, параметры в системе могут быть выбраны в следующем виде: $D_{p}=10^{-2}-10^{2} \mathrm{~cm}^{2} / \mathrm{s}, \quad n_{0} \gg 10^{21} \mathrm{~cm}^{-3}$, $G=10^{25}-10^{34} \mathrm{~cm}^{-3} / \mathrm{s}[1,17]$. Тогда для скорости фотогенерации свободных носителей с хорошей степенью точности подходит соотношение $[1,17]$

$$
G \approx \alpha I / \hbar \omega
$$

где $I$ - интенсивность света используемого лазерного источника, $\alpha-$ линейный коэффициент поглощения полупроводника (см. таблицу).

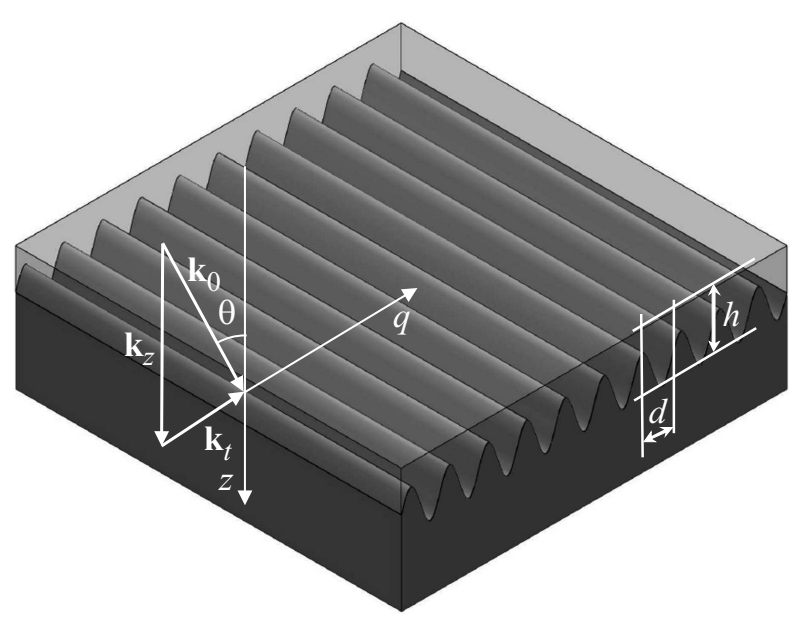

Рис. 2. Геометрия образования периодических структур в поле лазерного излучения. $\mathbf{k}_{0}-$ волновой вектор падающей волны ( $\mathbf{k}_{z}$ и $\mathbf{k}_{t}-$ его проекции); $\mathbf{q}-$ волновой вектор ППП (параллелен проекции $\mathbf{E}_{i}$ на плоскость поверхности). $d-$ период структуры, $h-$ глубина проникновения, $\theta-$ угол падения. 
Для коэффициента рекомбинации можно записать соотношение:

$$
\mu \cong 1 / \tau_{c}-\sigma_{c} I / \hbar \omega
$$

где $\tau_{c}$ - время рекомбинации (релаксации) зарядов, составляющее от $1 \mu \mathrm{s}$ до $1 \mathrm{ps} ; \sigma_{c}-$ сечение поглощения на отдельном носителе, составляющее порядка $10^{-16}-10^{-18} \mathrm{~cm}^{2}$.

В настоящей работе мы рассматриваем модель образования металлической пленки на поверхности облучаемого полупроводника. Однако, важно заметить что полной металлизации достичь не удается. При высоких концентрациях неравновесных носителей важную роль начинает играть Оже-рекомбинация, которую можно определить как $\mu_{n l} \simeq C n^{2}-$ где для кремния $C \simeq 4 \cdot 10^{-31} \mathrm{~cm}^{6} / \mathrm{s}$ [17]. Соответствующий эффект накладывает „границу сверху“ на концентрацию неравновесных носителей на поверхности полупроводника, что составляет приблизительно $n_{c} \simeq 10^{20}-10^{21} \mathrm{~cm}^{-3}$ при том, что полная концентрация валентных электронов в кремнии есть $n_{p} \simeq 5 \cdot 10^{23} \mathrm{~cm}^{-3}$.

Таким образом, скорость линейной рекомбинации неравновесных зарядов $\mu$ определяется разностью величины скорости генерации неравновесных носителей на поверхности полупроводниковой структуры за вычетом параметра, характеризующего скорость отрицательной рекомбинации (определяемой эффектом локального усиления поля на дефектах, а в нашем случае - на генерируемых неравновесных носителях). Показано, что в периодически модулированном по интенсивности световом поле происходит усиливаемое диффузионной неустойчивостью сверхбыстрое (пико- и субпикосекундной) образование локальных концентраций неравновесных носителей, что приводит к неоднородному нагреву металлизированной пленки, и соответствующий процесс носит резонансный характер. Как видно из (19), скорость этого дифузионного усиления

$$
g \sim q^{2} \sim 1 / d^{2} \sim 1 / h^{2} .
$$

Соответствующий механизм может быть реализован в условиях облучения поверхности полупроводника как непрерывным, так и импульсным лазерным излучением, причем во втором случае при использовании последовательностей ультракоротких лазерных импульсов [44-53] этот механизм выражен наиболее ярко.

Как видно из соотношений (15), усиление диффузионной неустойчивости происходит в том случае, если коэффициент рекомбинации становится отрицательным

$$
\mu \cong 1 / \tau_{c}-\sigma_{c} I / \hbar \omega<0,
$$

но при этом эффективный коэффициент генерации неравновесных носителей остается положительным

$$
G_{e f} \cong \alpha I / \hbar \omega+n_{0}\left(1 / \tau_{c}-\sigma_{c} I / \hbar \omega\right)>0 .
$$

Из (23) видно, что при критической интенсивности лазерного излучения

$$
I_{c}+\cong \frac{\hbar \omega n_{0}}{\tau_{c}} \frac{1}{n_{0} \sigma_{c}-\alpha}
$$

реализуется режим резонансного „сверхбыстрого“ усиления (при $\sigma_{c} n_{0}>\alpha$ ), когда параметр $\left|G_{e f}\right| \rightarrow 0$ и, следовательно, параметр, связанный с диффузионным усилением, достигает огромных значений $\left|g_{D}\right| \rightarrow \infty$. Кроме того, критическое значение интенсивности $I_{c}$ является значением, вблизи которого поведение системы может качественно изменяться.

С учетом характерных значений соответствующих параметров значение резонансной интенсивности можно оценить в интервале значений $10^{6}-10^{10} \mathrm{~W} / \mathrm{cm}^{2}$, соответствующих уровню мощностей доступных лазерных источников.

Так, при $I<I_{c}$, но при $I>\hbar \omega / \sigma_{c} \tau_{c}$, имеет место диффузионное усиление поля неравновесных зарядов. В области $\left|I_{c}-I\right| \rightarrow 0$ (но при $I<I_{c}$ ) наблюдается исключительно сильное усиление этого поля, а при $I>I_{c}-$ его резкое затухание. Отсюда видно, что в области критической интенсивности $I_{c}$ динамика системы может кардинальным образом изменяться при незначительном изменении мощности лазерного источника. Ясно, что в общем случае для анализа поведения системы в этой узкой области значений интенсивности излучения необходимо учитывать нелинейные эффекты более высокого порядка (прежде всего, влияние Ожерекомбинации, имеющей кубическую зависимость от концентрации неравновесных зарядов [17]).

Из соотношения (18) следует, что усиление генерируемой периодической структуры тем сильнее, чем меньше период наведенной на поверхности ППС. Заметим, что в первом приближении период ППС, возникающей между полупроводником и наведенной пленкой металлизации, приблизительно равен толщине последней $[11,17]$. Толщину фотоиндуцированной пленки металлизации по порядку величины можно считать обратно пропорциональной величине эффективного поглощения полупроводника, т.е. с хорошей степенью точности можно считать $q \approx 1 / h \approx \alpha$.

Тогда решение (19) можно переписать в виде

$$
g \approx-\left(\frac{1}{\tau_{c}}-\frac{\sigma_{c} I}{\hbar \omega}\right)\left(1+\frac{\alpha^{2} n_{0} D_{p}}{\frac{\alpha I}{\hbar \omega}+n_{0}\left(\frac{1}{\tau_{c}}-\frac{\sigma_{c} I}{\hbar \omega}\right)}\right) .
$$

Точный расчет толщины пленки металлизации $h$ и параметра $\alpha$, характеризующего глубину проникновения лазерного излучения в полупроводник, представляется сложной задачей, поскольку в общем случае глубина проникновения светового поля изменяется при увеличении концентрации свободных носителей. Кроме того, в условиях большой концентрации неравновесных носителей значительную роль начинает играть быстрая Ожерекомбинация носителей [17]. Также следует учесть, что 


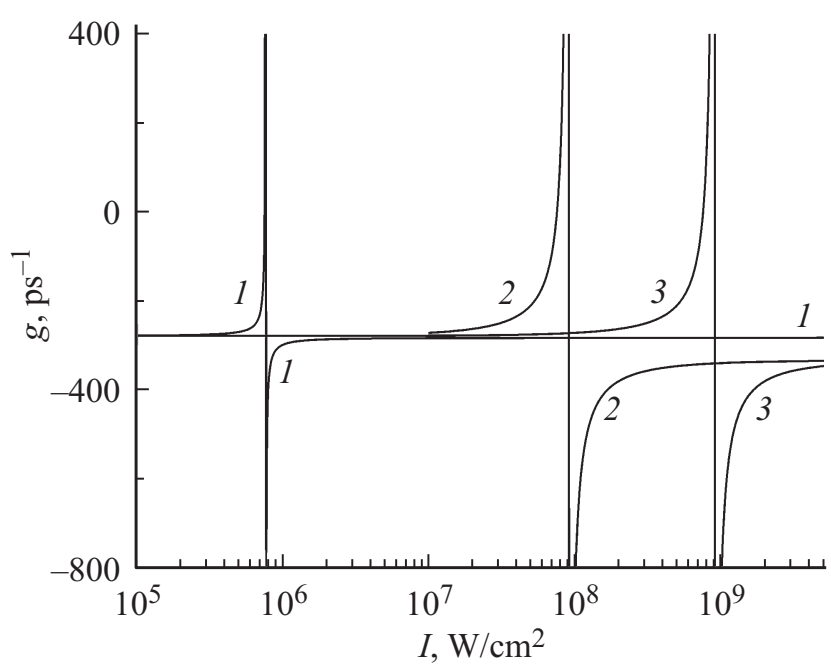

Рис. 3. Зависимость эффективного инкремента усиления от интенсивности лазера $g(I)$ для различных условий формирования ППП: $1-\tau_{c}=10^{-7} \mathrm{~s}, \lambda=265 \mathrm{~nm}, \alpha=6 \mathrm{~nm}^{-1}$, $n_{0}=0.168 \cdot 10^{29}, D_{p}=10^{-4}, \sigma_{c}=10^{-20} \mathrm{~m}^{2} ; 2-\tau_{c}=10^{-7} \mathrm{~s}$, $\lambda=265 \mathrm{~nm}, \quad \alpha=6 \mathrm{~nm}^{-1}, \quad n_{0}=0.168 \cdot 10^{29}, \quad D_{p}=10^{-4}$, $\sigma_{c}=10^{-20} \mathrm{~m}^{2} ; 3-\tau_{c}=10^{-6} \mathrm{~s}, \lambda=265 \mathrm{~nm}, \alpha=6 \mathrm{~nm}^{-1}$, $n_{0}=10^{30}, D_{p}=10^{-3}, \sigma_{c}=10^{-20} \mathrm{~m}^{2}$.

параметр $q$, определяемый соотношением (18), может значительно отличаться от величины $1 / h$ и, как следствие, от глубины проникновения $\alpha$.

Возможность варьирования интенсивности используемого излучения позволяет с большой точностью настраиваться на нужный диапазон резонансного диффузионного усиления (достигая гигантских значений $g \gg 10^{11} \mathrm{c}^{-1}$ ) и обеспечить сверхбыстрое (субпикосекундное) формирование неоднородного поля неравновесных носителей.

На рис. 3 представлены зависимости $g(I)$ (кривые $1-4)$, построенные на основе соотношения (25) с параметрами среды и излучения, подобранными таким образом, чтобы выполнялось условие (15.1). Из рисунка видно, что при определенных параметрах облучения поверхности (интенсивности лазерного облучения) приведенные зависимости могут носить резонансный характер.

Кроме того, следует отметить, что наибольшего усиления периодически модулированной плотности неравновесных зарядов (и, как следствие, высоты рельефа поверхности облучаемого полупроводника) можно добиться в условиях реализации медленных и сверхмедленных плазмон-поляритонных волн соответствующих наибольшим значениям $q$, что хорошо соотносится с имеющимися экспериментальными данными [14-16,31,36]. Таким образом, в случае генерации индуцированной медалической пленки на поверхности полупроводника образуется медленный плазмон-поляритон, характеризуемый волновым параметром $q$ из (18) и фазовой скоростью $v_{f}$. При этом волновое число соответствующего плазмонполяритона может достигать очень больших значений, значительно превышающих $1 / h$, и достигать в оптическом диапазоне величин $\sim 10^{8} \mathrm{~m}^{-1}$ [38-43]. Важно, что в этом случае соответствующее волновое число $q \gg \alpha$. Как следствие, диффузионное усиление в широком диапазоне значений может обеспечить генерацию и эффективное усиление периодической высококонтрастной структуры за время значительно меньшее 1 пикосекунды.

При этом в стандартных условиях $n_{0} \rightarrow \infty$ как в случае растущей диффузии, так и в случае спадающей диффузии, когда диффузионной нелинейностью можно пренебречь, диффузионная составляющая инкремента услиления стремится к значению $g_{D}^{+} \cong g_{D}^{-} \cong-q^{2} D_{0}$ и всегда остается отрицательной, что способствует скорейшему затуханию соответствующих интерференционных компонент - возмущений (что особенно актуально в условиях больших значений коэффициента диффузии).

В заключение нужно отметить, что к настоящему времени нет точного представления (во всяком случае, у авторов настоящей работы) о величине параметра $n_{0}$, определяющего влияние нелинейности диффузии, и даже о его знаке. Более того, вопрос о характере движения фотовозбужденной электронно-дырочной плазмы в целом, несмотря на более чем давнюю историю [52-54], и по сей день остается открытым. Так, зафиксированы наблюдения, указывающие на удержание плазмы вблизи поверхности полупроводников в поле лазерного излучения. В этом случае логично сделать вывод об уменьшении диффузии при увеличении концентрации зарядов, т. е. справедлив сценарий (15.1). С другой стороны, логично предположить, что области с повышенной концентрацией зарядов будут иметь большую локальную температуру и, как следствие, в этих точках диффузия усиливается по сравнению с областями с меньшей концентрацией, т. е. справедлив сценарий (15.2).

Вместе с тем, как было показано выше, скорость генерации поверхностной дефектной структуры в поле медленного плазмон-поляритона вблизи резонансных значений усиления может достигать гигантских значений порядка $g_{D}=10^{11}-10^{14} \mathrm{~s}^{-1}$, и здесь можно говорить о диффузионном „взрыве“, приводящем к сверхбыстрому образованию высококонтрастной периодической структуры за субпикосекундные промежутки времени.

\section{4. Заключение}

В рамках предложенной модели нелинейной диффузии обнаружен сильный резонансный эффект, способный приводить к сверхбыстрому пико- и субпикосекундному усилению генерируемой на поверхности плазмонполяритонной структуры. В этом случае может быть получена высококонтрастная решетка дефектов, разрастающаяся за время, значительно меньшее пикосекунды. Процесс образования высококонтрастной периодической структуры можно представить следующим образом $[1,17]$.

1. Процесс начинается с возникновения периодически модулированного интерференционного светового поля падающего лазерного излучения и ППП. 

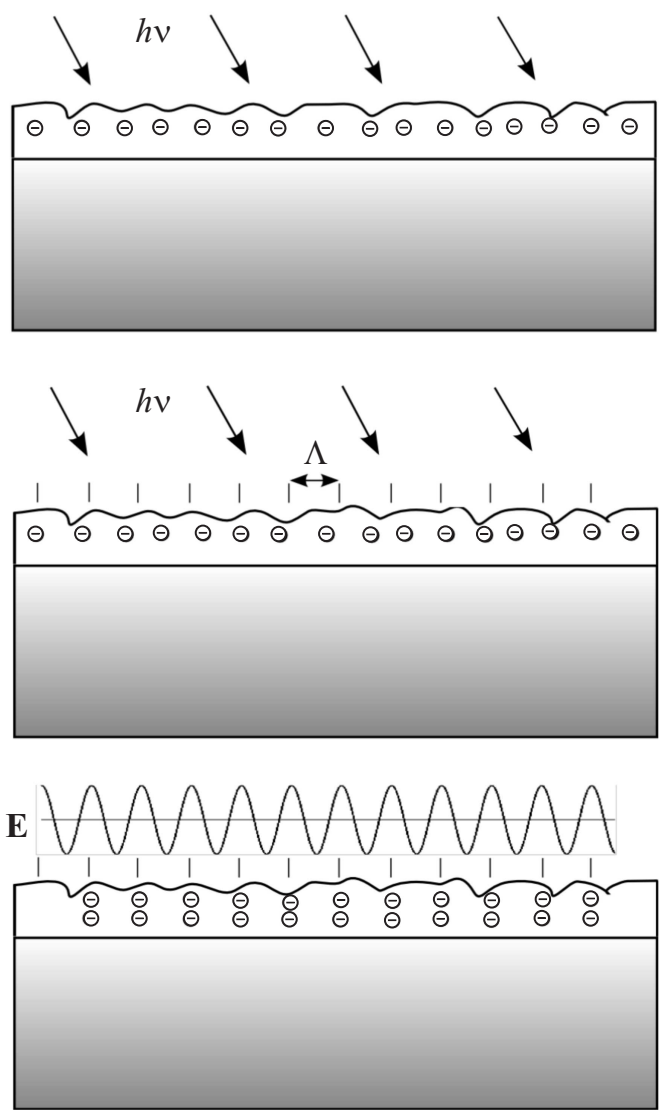

Рис. 4. Возникновение периодического рельефа на поверхности облучаемого полупроводника в поле поверхностного плазмон-поляритона.

2. В периодически модулированном по интенсивности световом поле происходит, как нами показано, усиливаемое диффузионной неустойчивостью сверхбыстрое (пико- и субпикосекундное) образование локальных концентраций неравновесных носителей, что приводит к неоднородному нагреву возникающей металлизированной пленки. Показано, что соответствующий процесс носит резонансный характер.

3. При достаточной мощности лазерного излучения неоднородный нагрев вызывает неоднородное плавление и сверхбыстрый вынос вещества с сохранением интерференционного рельефа по окончании облучения.

Соответствующие „этапы“ наноструктурирования поверхности в поле генерируемого лазерным излучением плазмон-поляритона представлены на рис. 4.

4. Одновременно с этим реализуется сценарий возникновения неустойчивости за счет положительной обратной связи по следующей схеме. Появление микрорельефа поверхности с определенными периодом и фазой способствует повышенному поглощению в локальных максимумах микрорельефа, что еще более увеличивает глубину модуляции температуры и приводит к дальнейшему повышению поглощения и т. д. При этом время генерации высококонтрастной периодической структуры с большой амплитудой неоднородности может быть значительно меньше 1 пикосекунды.

Представляется, что именно обнаруженное специфичное усиление возмущений в системах с нелинейной диффузией может быть механизмом, обеспечивающим запись высококонтрастных периодических рельефных структур микро- и нанометрового масштабов.

Предложенная нами модель оптимальным образом может быть использована для получения поверхностных когерентных структур при использовании лазерных источников с интенсивностями $I_{\max }<10^{12} \mathrm{~W} / \mathrm{cm}^{2}$, т. е. до тех значений, при которых начинается процесс сильного выброса (абляции) вещества, связанного с образованием так называемой эрозионной лазерной плазмы.

\section{Список литературы}

[1] С.А. Ахманов, В.И. Емельянов, Н.И. Коротеев, В.Н. Семиногов. УФН 147, 675 (1985).

[2] A.M. Bonch-Bruevich, M.N. Libenson. Nonlin. Electromagn. Surf. Phenomena / Ed. H.-E. Ponath, G.I. Stegeman. North Holland, The Netherlands (1991).

[3] A.M. Bonch-Bruevich, M.N. Libenson, V.S. Makin, V.V. Trubaev. Opt. Eng. 31, 716 (1992).

[4] В.И. Емельянов, И.М. Панин. ФТТ 39, 2029 (1997).

[5] В.И. Емельянов, А. Байдуллаева, А.И. Власенко, П.Е. Мозоль. Квант. электроника 38, 245 (2008).

[6] В.В. Баженов, А.М. Бонч-Бруевич, М.Н. Либенсон, В.С. Макин. Письма в ЖТФ 10, 20, 1520 (1984).

[7] Е.В. Голосов, В.И. Емельянов, А.А. Ионин, Ю.Р. Колобов, С.И. Кудряшов, А.Е. Лигачев, Ю.Н. Новоселов, Д.В. Селезнев, Д.В. Синицын. Письма в ЖЭТФ 90, 2, 16 (2009).

[8] S. Sakabe, M. Hashida, S. Tokita, S. Namba, K. Okamuro. Phys. Rev. B 79, 033409 (2009).

[9] A. Welch, T.H.R. Crawford, D.S. Wilkinson, H.K. Haugen, J.S. Preston. Appl. Phys. A 89, 1001 (2007).

[10] В.С. Макин, Ю.И. Пестов, Р.С. Макин, А.Я. Воробьев. Опт. журн. 76, 9, 38 (2009).

[11] J. Bonse, M. Munz, H. Sturm. J. Appl. Phys. 97, 013538 (2005).

[12] M.G. Couillard, A. Borowiec, H.K. Haugen, J.S. Preston, E.M. Griswold, G.A. Botton. J. Appl. Phys. 101, 033519 (2007).

[13] A. Borowiec, H.K. Haugen. Appl. Phys. Lett. 42, 25, 4462 (2003).

[14] С.В. Заботнов, Л.А. Головань, И.А. Остапенко, Ю.В. Рябчиков, А.В. Червяков, В.Ю. Тимошенко, П.К. Кашкаров, В.В. Яковлев. Письма в ЖЭТФ 83, 2, 76 (2006).

[15] A.Y. Vorobyev, V.S. Makin, C. Guo. J. Appl. Phys. 101, 034903 (2007).

[16] P. Berini, I. De Leon. Nature Photon. 6, 16 (2012).

[17] Н.И. Коротеев, И.Л. Шумай. Физика мощного лазерного излучения. Наука, М. (1991). С. 309.

[18] М.Н. Либенсон, А.М. Бонч-Бруевич, В.С. Макин. УФН 155, 719 (1988).

[19] А.А. Самарский, А.П. Михайлов. Математическое моделирование. (Идеи. Методы. Примеры). Физматлит, М. (2005). $320 \mathrm{c}$.

[20] А.А. Самарский, В.А. Галактионов, С.П. Курдюмов, А.П. Михайлов. Режимы с обострением в задачах для квазилинейных параболических уравнений. Наука, М. (1987). $477 \mathrm{c}$. 
[21] С.Н. Аристов. ПМТФ 40, 22 (1999).

[22] В.М. Журавлев. Письма в ЖЭТФ 65, 285 (1997).

[23] В.М. Журавлев. ЖЭТФ 114, 1897(1998).

[24] В.М. Журавлев. ТМФ 124, 265 (2000).

[25] В.В. Климов. Наноплазмоника. Физматлит, М. (2009). $480 \mathrm{c}$.

[26] Поверхностные поляритоны. Электромагнитные волны на поверхностях и границах раздела сред / Под ред. В.М. Аграновича, Д.Л. Миллса. Наука, М. (1985). 526 с.

[27] А.М. Бонч-Бруевич, М.К. Коченгина, М.Н. Либенсон, В.С. Макин, С.Д. Пудков, В.В. Турбаев. Изв. АН СССР. Сер. физ. 46, 1186 (1982).

[28] A.M. Bonch-Bruevich, M.N. Libenson, V.S. Makin, V.V. Turbaev. Opt. Eng. 31, 4, 718 (1992).

[29] В.С. Макин, Р.С. Макин. Основы взаимодействия ультракороткого лазерного излучения с конденсированными средами. Димитровград (2013). С. 236.

[30] А.Н. Тихонов, А.А. Самарский. Уравнения математической физики. Наука, М. (1972). 73 с.

[31] E.N. Economou. Phys. Rev. 182, 539 (1969).

[32] J.J. Burke, G.I. Stegeman, T. Tamir. Phys. Rev. B 33, 5186 (1986).

[33] M.N. Zervas. Opt. Lett. 16, 720 (1991).

[34] D. Sarid. Phys. Rev. Lett. 47, 1927 (1981).

[35] P. Berini. Opt. Lett. 24, 1011 (1999).

[36] A.V. Zayats, I.I. Smolyaninov, A.A. Maradudin. Phys. Rep. 408, 131 (2005).

[37] Д.Ю. Федянин, А.В. Арсенин, В.Г. Лейман, А.Д. Гладун. Квант. электроника 39, 8, 745 (2009).

[38] С.В. Жирнов, Д.И. Семенцов. ФТТ 49, 773 (2007)

[39] Д.Г. Санников, С.В. Жирнов, Д.И. Семенцов. ФТТ 51, 1824 (2009)

[40] Д.Г. Санников, А.С. Абрамов, И.О. Золотовский, Д.И. Семенцов. ФТТ 57, 4, 639 (2015).

[41] И.О. Явтушенко, М.С. Явтушенко, И.О. Золотовский С.Г. Новиков, А.В. Беринцев, А.С. Кадочкин, Д.А. Столяров, Б.Б. Костюшко, Н.А. Бунаков. Письма в ЖТФ 41, 60 (2015).

[42] A.A. Ionin, S.I. Kudryashov, S.V. Makarov, L.V. Seleznev, D.V. Sinitsyn, E.V. Golosov, O.A. Golosova, Yu.R. Kolobov, A.E. Ligachev. Appl. Phys. A 107, 301 (2012).

[43] M. Huang, F. Zhao, Y. Cheng, N. Xu, Z. Xu. Opt. Express 16, 23, 19354 (2008).

[44] J. Bonse, A. Rosenfeld, J. Kruger. J. Appl. Phys. 106, 104910 (2009).

[45] T. Tomita, K. Kinoshita, S. Matsuo, S. Hashimoto. Appl. Phys. Lett. 90, 153115 (2007).

[46] R. Le Harzic, H. Schuck, T. Anhut, I. Riemann. Opt. Express 13, 17, 6651 (2005).

[47] A. Borowiec, H. Haugen. Appl. Phys. Lett. 83, 25, 4462 (2003).

[48] B. Tan, K. Venkatakrishnan. J. Micromech. Microeng. 16, 1080 (2006).

[49] X. Wang, G. Lim. Surf. Rev. Lett. 12, 4, 651 (2005).

[50] H. Quyang, Y. Deng, W. Knox, P.M. Fauchet. Phys. Status Solidi B 204, 5, 1255 (2007).

[51] E. Hsu, T. Crawford, C. Manders, G.A. Botton, H.K. Haugen. Appl. Phys. Lett. 92, 221112 (2008).

[52] A. Forchel, B. Laurich, H. Hillmer. J. Luminescence 30, 67 (1985).

[53] J.P. Wolfe. J. Luminescence 30, 82 (1985).

[54] H. Schweizer, E. Zilinski, A. Forchel, G. Mahler. J. Luminescence 31, 503 (1986). 